\title{
Resonance Decays Involving Two Partial Waves
}

\author{
W. P. PETERSEN* \\ $b^{4}$ University of Minnesota, Minneapolis, Minnesota, 55455 \\ and
}

J. L. ROSNER ${ }^{\dagger}$

California Institute of Technology, Pasadena, California, 91109

February, 1972

\begin{abstract}
Resonance decays involving two partial waves, such as $B \rightarrow \omega \pi$, can test for basic violations of $\mathrm{SU}(6)_{\mathrm{W}}$. A formalism is presented for carrying out these tests using available data on $\pi \mathrm{N} \rightarrow \pi \Delta(1236)$ and $\mathrm{K}^{-} \mathrm{p} \rightarrow \pi \mathrm{Y}_{1}{ }^{*}(1385)$.
\end{abstract}

*Supported in part by the U.S. Atomic Energy Commission under Contract AT-(11-1)-1764. tSupported in part by the U.S. Atomic Energy Commission under Contract AT- $(11-1)-68$. Alfred P. Sloan Foundation Fellow. Permanent Address: School of Physics and Astronomy, University of Minnesota, Minneapolis, Minnesota 55455.

This report was prepared as an account of work sponsored by the United States Government. Neither Commited States nor the United States Atomic Energy Commission, nor any of their employees, nor any of their contractors, subcontractors, or their employees, legal liability or ranty, express or implied, or assumes any pleteness or or responsibility for the accuracy, comproduct or process disclosed, information, apparatus, would not infringe privately owned rights. 


\section{DISCLAIMER}

This report was prepared as an account of work sponsored by an agency of the United States Government. Neither the United States Government nor any agency Thereof, nor any of their employees, makes any warranty, express or implied, or assumes any legal liability or responsibility for the accuracy, completeness, or usefulness of any information, apparatus, product, or process disclosed, or represents that its use would not infringe privately owned rights. Reference herein to any specific commercial product, process, or service by trade name, trademark, manufacturer, or otherwise does not necessarily constitute or imply its endorsement, recommendation, or favoring by the United States Government or any agency thereof. The views and opinions of authors expressed herein do not necessarily state or reflect those of the United States Government or any agency thereof. 


\section{DISCLAIMER}

Portions of this document may be illegible in electronic image products. Images are produced from the best available original document. 
When a meson or baryon resonance decays into two particles at least one of which has sufficiently high intrinsic spin, these two particles can appear in more than one partial wave. The helicity distribution of the final particles will then depend on the relative phase and magnitude of the ampltiudes in these different partial waves. The most familiar case is the decay $B \rightarrow \omega \pi$, in which the helicity distribution of the final $\omega$ indicates that both $S$ and $D$ waves are present. ${ }^{1)}$

The ratio of such amplitudes is of interest for several reasons:

(1) A definite value for it is predicted by $\left.S U(6)_{W} \times 0(2)_{L_{z}} \cdot 2,3\right)$ This symmetry is found to be violated in a way not explicable by centrifugal barrier effects alone. ${ }^{1)}$

(2) It is the only free parameter by which $\mathrm{SU}(6)_{\mathrm{W}} \times 0(2)_{\mathrm{L}_{\mathrm{Z}}}$ is modified in certain recent pictures of hadron decays. ${ }^{1,4-7)}$.

(3) In relativistic quark models it is parametrized by "quark recoil effects". These effects have been shown essential to a correct description of hadronic decays and photoproduction. ${ }^{6,7)}$ Models not incorporating such effects ${ }^{8,9}$ ) fix this ratio at the value predicted by $\left.\mathrm{SU}^{(6)}\right)_{\mathrm{W}} \times 0(2){ }_{\mathrm{L}_{\mathrm{Z}}}$.

(4) It is now experimentally accessible in a number of cases involving baryon decays, particularly, into $\Delta(1236) \pi$ and $Y_{1}^{*}(1385) \pi$. We shall show how baryon decays tell the sign of the amplitude ratio, and what progress has been made to date. The subject is of present experimental interest because of recent studies of $\pi N \rightarrow \pi \pi N^{11)}$ and $K-N \rightarrow \pi \pi \Lambda .^{12,13)}$ Detalls of more theoretical interest will be presented elsewhere ${ }^{14)}$.

Consider decays involving a pion or kaon and a "ground state" baryon:

$$
A\left(J^{A}, L ; S^{A} ; \lambda\right) \rightarrow B\left(J^{B}, L=0, S^{B}=J^{B} ; \lambda\right)+C\left(J^{C}=0\right)
$$

Here $L$ and $S$ refer to quark orbital and spin angular momenta. 
Let the z-axis be the direction of $B$, and $\lambda$ the projection of $\mathrm{J}^{\mathrm{A}}$ along this axis. Assign $A, B$, and $C$ to multiplets of static SU(6) $\times 0(3)$. Then take the decay to proceed via the creation of a $q \bar{q}$ pair, one of whose members emerges in each final hadron. The pair is assumed to have the quantum numbers of the vacuum: $\mathrm{SU}(3)$ singlet, $\mathrm{J}^{\mathrm{PC}}=0^{++}$(i.e. $\left.{ }^{3} \mathrm{P}_{0}\right) .{ }^{1,3-5)}$ This language, motivated by duality graphs, 15,4$)$ may be more valid than the quark model on which it is based. ${ }^{16)}$

The decay helicity amplitudes are then written in our model as

$$
M^{(\lambda)}(A \rightarrow B C)=\sum\left(\begin{array}{c|cc}
A & B & 35 \\
\alpha, a & \beta, b & 8,3
\end{array}\right) i\left(\begin{array}{l|ll}
\alpha & \beta & 8 \\
A & B & C
\end{array}\right) i X_{\lambda}(A \rightarrow B C)
$$

where

$$
\mathrm{X}_{\lambda}(\mathrm{A} \rightarrow \mathrm{BC})=\sum_{\mathrm{L}_{z}}\left(\mathrm{~J}^{\mathrm{B}} \dot{\lambda} 1-\mathrm{L}_{z} \mid \mathrm{S}^{\mathrm{A}} \lambda-\mathrm{L}_{z}\right)\left(\mathrm{S}_{\lambda-\mathrm{L}_{z}}^{\mathrm{A}} \mathrm{L} \mathrm{L}_{z} \mid \mathrm{J} \lambda\right) \mathrm{a}_{\mathrm{L}}\left(\mathrm{L}_{z}\right)
$$

In Eq. (2) the first term is an SU(3)-scalar factor, ${ }^{17)}$ with $\underset{m}{\mathrm{~A}}, \mathrm{~m}$ and 35 labeling SU(6) representations and $\alpha, a ; \beta, b ;$ and 8,3 labeling SU(3) $x$ SU(2) representations. The second term is an isoscalar factor ${ }^{18)}$ with $A, B$, and $C$ labeling specific isomultiplets. The sum over $\underline{i}$ corresponds to $d$ and $f$ coupling when $\alpha$ and $\beta$ are octets. For decays into specific charge states $M^{(\lambda)}$ is to be multiplied by an appropriate Clebsch-Gordan coefficient.

In Eq. (2) and ${ }^{3} \mathrm{P}_{0}$ "spurion" has been combined with the pseudoscalar meson to form an effective $(8,3)$ member of the 35 of $\mathrm{SU}(6)$. For baryon decays the formalism is thus manifestly equivalent to $\mathrm{SU}(6)_{\mathrm{W}} \times 0(2)_{L_{z}}$ when one takes $a_{L}( \pm 1)=0$. This is so for meson decays as we11. 3,14$)$

The first Clebsch-Gordan coefficient in Eq. (3) describes quark spin conservation while the second describes the coupling of quark spin and quark $\mathrm{L}$ to $\mathrm{J} \equiv \mathrm{J}^{\mathrm{A}}$. The reduced matrix elements $\mathrm{a}_{\mathrm{L}}\left(\mathrm{L}_{\mathrm{z}}\right)$ are assumed to depend only on 
$\mathrm{L}_{z}$ and on the specific $\mathrm{SU}(6) \times O(3)$ multiplets involved in the decay $\mathrm{A}(\mathrm{L}) \rightarrow \mathrm{B}$ C.

The rule derived in Ref. 1 relating the decays $A_{1} \rightarrow \rho \pi$ and $B \rightarrow \omega \pi$, namely $2\left(x_{1} / x_{0}\right)_{A}=\left(x_{0} / x_{1}\right)_{B}+1$, then follows directly from $E q .3$.

In our normalization the partial width is

$$
\Gamma(A \rightarrow B C)=\frac{1}{2 J^{A}+1} \frac{p}{M_{A}^{2}} \sum_{\lambda}\left|M^{(\lambda)}(A \rightarrow B C)\right|^{2,}, \vdots
$$

where $\mathrm{p}$ is the magnitude of the final CM 3-momentum.

Symmetry breaking due to centrifugal barrier terms is best described in terms of reduced matrix elements labeled by the partial wave $\ell=L \pm 1$ of the final state. We shall define amplitudes $a_{L}^{(l)}$ normalized so that when $a_{L}( \pm 1)=$ $0, a_{L}{ }^{(L+1)}=a_{L}{ }^{(L-1)}=a_{L}{ }^{(0)}$. Then since $L$ and the spurion angular momentum make up the final orbital angular momentum $\ell=L \pm 1$, we have

$$
\underline{a}_{L}^{(\ell)}=\left(\begin{array}{lllllll}
L & 0 & 1 & 0 & \ell & 0
\end{array}\right)^{-1} \sum_{L_{z}}\left(\begin{array}{llllll}
L_{z} & L_{z} & 1 & -L_{z} \mid \ell & 0
\end{array}\right) a_{L}\left(L_{z}\right) .
$$

The inverse of (5) allows one to write Eq. (3) as

$$
\mathrm{x}_{\lambda}=\sum_{\ell=\mathrm{L} \pm 1}(2 \ell+1)^{1 / 2}\left(\mathrm{~J}^{\mathrm{B}} \lambda \ell 0 \mid \mathrm{J} \lambda\right) \gamma_{\ell-I_{L}}^{(\grave{l})} \text {, }
$$

where $\left.\gamma_{\ell} \equiv(2 \mathrm{~S}+1)^{1 / 2}(\mathrm{~L} 010 \mid \ell \quad 0) \quad \mathrm{W}^{\mathrm{B}} \mathrm{J}^{\mathrm{B}} 1 \mathrm{~J} \cdot \mathrm{L} ; \mathrm{S}^{\mathrm{A}} \ell\right)$.

The normalization in Eq. (6) is appropriate since in calculating partial widths we use the quantity

$$
\frac{1}{2 \mathrm{~J}+1} \sum_{\lambda}\left|\mathrm{X}_{\lambda}\right|^{2}=\left.\sum_{\ell=\mathrm{L} \pm 1} \gamma_{\ell}{ }^{2} \underline{\underline{a}}_{\mathrm{L}}(\ell)\right|^{2}
$$

In Eq. (7) $W\left(J^{B} 1 \mathrm{~J} L ; S^{A} l\right)$ is a Racah coefficlent as defined, for example, in Edmonds. 20) 
The coefficients $\underline{a}_{L}^{(\ell)}$ are taken to be universal for decays within a given multiplet, aside from a centrifugal barrier term. For this term we take a zero-radius form, 1,5

$$
\underline{a}_{L}^{(l)}=\tilde{a}_{L}^{(l)}\left(p \mid p_{o}\right)^{\ell}
$$

and define a scale for $a_{L}(l)$ by seting $p_{0}=0.5 \mathrm{GeV}$ as in Ref. 1 .

Preliminary fits to the decays of $(35, L=1)$ mesons 1 and $(20, L=1)$

and $(56, L=2)$ baryons yield reasonably self-consistent values of $\left|a_{L}(\ell)\right|$ in all three cases. (See Tables I- - III). These cases share the property that

$$
\left|\mathrm{a}_{\mathrm{L}}(\mathrm{L}+1)\right| \simeq\left|\mathrm{a}_{\mathrm{L}}(\mathrm{L}-1)\right|
$$

As a large number of the decays considered have $\mathrm{p} \simeq 0.5 \mathrm{GeV}$, this indicates (modulo symmetry breaking due to mass differences) that $\left|\underline{a}_{L}(L+1)\right| \simeq\left|\underline{a}_{L}(L-1)\right|$. The $S U(6){ }_{W} \times O(2){ }_{L_{z}}$ symmetric case is $\underline{a}_{L}(L+1)=\underline{a}_{L}(L-1)$. On the other hand, for $L=1$ meson decays one finds $\tilde{a}_{L}(L+1) \simeq-\tilde{a}_{L}(L-1)$, as a result of the dominantly transverse nature of the $B \rightarrow \omega \pi$ decay. ${ }^{1)}$

It is clearly of interest to determine when such a sign change occurs, and hence when $S U(6){ }_{W} \times O(2)_{L_{z}}$ is breaking down in a manner more fundamental than would follow from barrier effects alone. We list some examples whereby this may be learned from baryon decays.

a. Reaction $\underline{\pi N \rightarrow \pi \Delta}$. Partial-wave analyses of $\pi^{-} p \rightarrow \pi^{+} \Delta^{-}$in the range $E_{\mathrm{CM}}=1.5$ to $1.7 \mathrm{GeV}$ can extract the relative signs of various amplitudes for decay into a ground state baryon and meson. Let us call

$$
\begin{aligned}
& \mathrm{S} \equiv \underline{a}_{\mathrm{L}=1}^{(l=0)} \quad(70 \rightarrow 5635) ; \mathrm{D} \equiv \underline{a}_{\mathrm{L}=1}^{(l=2)} \quad(70 \rightarrow 56 \underset{m}{35}) \\
& P \equiv \underline{a}_{L=2}^{(l=1)}(56 \rightarrow 56 \underset{\sim}{35}) ; \dot{F} \equiv \underline{a}_{L=2}^{(l=3)}(\underset{\sim}{56} \rightarrow 56 \text { 35) }
\end{aligned}
$$


The dominant $\Delta \pi$ resonances in this region are the $D_{13}(1520), D_{15}(1670)$, and $\mathrm{F}_{15}(1690)$. Their decays are given in the present model by:

$$
\begin{aligned}
& M^{(\lambda=1 / 2)}\left(D^{13} \rightarrow N \pi\right)=D / 3 \sqrt{3} \\
& M^{(\lambda=1 / 2)}\left(D^{13} \rightarrow \Delta \pi\right)=-(S+D) / 3 \sqrt{6} \\
& M^{(\lambda=3 / 2)}\left(D^{13} \rightarrow \Delta \pi\right)=-(S-D) / 3 \sqrt{6} \text {. } \\
& M^{(\lambda=1 / 2)}\left(D^{15} \rightarrow N \pi\right)^{i}=-D / 2 \sqrt{30} \\
& M^{(\lambda=1 / 2)}\left(D^{15} \rightarrow \Delta \pi\right)=D / 2 \sqrt{15} \\
& M^{(\lambda=3 / 2)}\left(D^{15} \rightarrow \Delta \pi\right)=D / \sqrt{10} \\
& M^{(\lambda=1 / 2)}\left(F^{15} \rightarrow \mathrm{N} \pi\right)=-F / 3 \sqrt{2} \\
& M^{(\lambda=1 / 2)}\left(F^{15} \rightarrow \Delta \pi\right)=-(4 / 75)(3 P+2 F) \\
& M^{(\lambda=3 / 2)}\left(F^{15} \rightarrow \Delta \pi\right)=-(4 / 25)(2 / 3)^{1 / 2}(P-F)
\end{aligned}
$$

where we have taken the $D_{13}$ to belong to an $(8,2)$ of $(70, L=1)$, the $D_{15}$ to be $(8,4)$ of $(70, L=1)$, and the $F_{15}$ to be $(8,2)$ of $\left.(56, L=2) .6\right)$ Note that only $\ell=2$ is predicted for $D_{15} \rightarrow \Delta \pi$; the model forbids $\ell=4$.

To a first approximation we neglect $D$ in comparison with $S$ in Eqs. $(12 \mathrm{~b}, \mathrm{c})$ and $\mathrm{F}$ in comparison with $\mathrm{P}$ in Eqs. $(13 \mathrm{~b}, \mathrm{c})$, because of the large centrifugal barrier. Then one may write amplitudes due to these resonances as, for example,

$$
\begin{aligned}
M_{1 / 21 / 2}(\pi N \rightarrow \pi \Delta)= & {\left[-D^{2} B_{D}^{5 / 2}(\theta)+F P_{B} F^{5 / 2}(\theta)\right] d_{1 / 21 / 2}^{5 / 2}(\theta)+} \\
& -S D B_{D}^{3 / 2}(\theta) d_{1 / 21 / 2}^{3 / 2}(\theta)
\end{aligned}
$$

where $B_{D}^{5 / 2}(s), B_{F}^{5 / 2}(s)$, and $B_{D}^{3 / 2}(s)$ are appropriately normalized Breit-Wigner factors with positive imaginary parts. 
Eq. (15) shows that for $F P<0$, the $D_{15}$ and $F_{15}$ interfere constructively in $\mathrm{M}_{1 / 2 \mathrm{~L} / 2}$, while for $\mathrm{FP}>0$ they interfere destructively. Published phase shift solutions allow for both cases: FP $>0$ ("solution $\mathrm{A}^{\text {") }}$ ) and FP < 0 ("solution $\left.B^{\prime \prime}\right)$. (Solution $A$ is that of $S U(6)_{W} \times 0(2)_{L_{z}}$. ) Recent modifications of the analysis favor solution A. $^{21)}$

The relative sign of $\mathrm{D}_{13}$ and $\mathrm{F}_{15}$ or $\mathrm{D}_{15}$ contributions may also be compared with experiment. An early $\mathrm{fit}^{22}$ ) contained such information but has been superseded; 11 ) the analysis is still in progress.

b. Reaction $\mathrm{K}\urcorner \mathrm{N} \rightarrow \pi \mathrm{Y}_{1}{ }^{*}$. One can obtain the relative sign of the contributions of $\Sigma(1765)\left(5 / 2^{-}\right)$and $\Lambda(1815)\left(5 / 2^{+}\right)$by similar means. ${ }^{23)}$ In $\mathrm{M}_{1 / 2} 1 / 2$, we predict that for $F P>0, \Sigma(1765)$ and $\Lambda(1815)$ will interfere constructively in $\mathrm{K}^{-} \mathrm{P} \rightarrow \pi \mathrm{Y}_{1}{ }^{*+}$ and destructively in $\mathrm{K}^{-} \mathrm{P}^{+} \rightarrow \pi^{+} \mathrm{Y}_{1}^{*-}$, and vice versa for $\mathrm{FP}<0$. Experimentally the situation is not resolved as yet. An older fit ${ }^{12}$ ) supports FP $>0$, while a more recent one favors FP $<0 .{ }^{13)}$ This more recent work has the disturbing feature that the branching ratio for $\Lambda(1815) \rightarrow \pi \mathrm{Y}_{1}{ }^{*}$ is far too low to be consistent via SU(3) with even the lowest possible estimates ${ }^{11}$ ) of $N(1690) \rightarrow \pi \Delta$

c. Helicity structure in decays. Although $\mathrm{D} / \mathrm{S}$ in Eqs. $(12 \mathrm{~b}, \mathrm{c})$ and F/P in Eqs. $(14 \mathrm{~b}, \mathrm{c})$ are too small to change the sign of any of the helicity amplitudes, they can alter the relative $\mathrm{M}^{(3 / 2)} / \mathrm{M}^{(1 / 2)}$ proportions significantly. For the $D_{13} \pi N$ resonance our fits predict $M^{(3 / 2)} / M^{(1 / 2)} \simeq 2 / 3$ for $D / S>0$ (this is the $S U(6)$ sign) and $\simeq 3 / 2$ for $D / S<0\left(\operatorname{anti-SU(6))}\right.$. For $F_{15}$, these ratios are $\simeq 1 / 4$ for $F / P>0(S U(6)-1 i k e)$ and $\simeq 2$ for $F / P<0($ anti-SU(6)). Some theoretical support; based on duality, exists for the enhancement of the $\lambda=3 / 2$ couplings. These couplings are automatically enhanced for $D_{15} \rightarrow \Delta \pi: M^{(3 / 2)} / M^{(1 / 2)}=\sqrt{6}$. 
For any abnormal-parity resonance on the leading trajectory $\left(\mathrm{J}^{\mathrm{P}}=5 / 2^{-}, 7 / 2^{+}\right.$, $\left.9 / 2^{-}, \ldots\right)$ our model predicts only the partial wave $\ell=J-1 / 2$ to contribute to $\Delta \pi$ decay, with $M^{(3 / 2)} / M^{(1 / 2)}=\sqrt{3(\ell+2) / l}$. Note that strict $S U(6)_{W} \times 0(2) L_{z}$, i.e. $D=S$ and $F=P$, predicts complete suppression of the $\lambda=3 / 2$ couplings in Eqs. $(12 \mathrm{c})$ and $(14 \mathrm{c})$. This result follows most directly from $\mathrm{s}_{\mathrm{z}}$ conservation. ${ }^{25)}$ The above effects, particularly for the $F$-wave resonances, should be observable. We would argue against fixing $F / P$ a priori in analyses ${ }^{12)}$, and even more so against neglecting it altogether. ${ }^{13)}$

Mixing. When comparing our formalism with the data, one must allow for mixing among nearby states. of different $\mathrm{S}^{\mathrm{A}}$ or $\mathrm{SU}(3)$ or $\mathrm{SU}(6)$ representation. One hint that the $D_{13}(1520)$ is not strongly mixed is that (here) the $s^{A}=3 / 2$ $D_{13} \pi N$ resonance has $1 / 40$ the partial width into $\pi N$ (modulo phase space) of its $S^{A}=1 / 2$ partner. The experimental absence of a second $D_{13}$ resonance in $\pi N$ phase shifts ${ }^{28}$ ) then suggests that the two states are relatively pure in $\mathrm{s}^{\mathrm{A}}$. Our discussion (part a) regarding $\mathrm{SD} / \mathrm{D}^{2}$ is thus likely to be reliable. On the other hand, the $\mathrm{F}_{15}$ (1690) may not be a pure member of the $56, \mathrm{~L}=2$. The existence of a 70, $\mathrm{L}=2$ degenerate with the $56, \mathrm{~L}=2$ is suggested by the harmonic oscillator quark mode $1,8,9)$ by duality, and to some extent by the data. ${ }^{27)}$ If the two $\mathrm{F}_{15} \mathrm{~N}^{*}$ states in the $70, \mathrm{~L}=2$ were to cause the $\mathrm{F}_{15}(1690)$ to be appreciably'mixed, its $\Delta \pi$ decay would probably not have the simple significance noted here.

Sumary. We have introduced a scheme for describing hadron decays which avoids the link between two different partial waves characteristic of certain symmetry approaches, $2,3,8,9)$ but preserves all other relations of such approaches. We have described several crucial tests involving the helicity structure of baryon decays that would indicate whether avoiding this link is in fact important. remant

We thank R. Cashmore, A. Kernan, F. Ravndal, J. Mandula and A. Rosenfeld for useful discussions. J.R. thanks G. Zweig for extending the hospitality of Caltech. 


\section{REFERENCES}

1. E.W. Colglazier and J.L. Rosner, Nucl. Phys. B27, 349 (1971).

2. This has generally been considered the only viable candidate for a decay symmetry involving L-excited hadrons. See, e.g., P.G.O. Freund, A.N. Maheshwari, and E. Schonberg, Phys. Rev. 159, 1232 (1967); H.J. Lipkin, Ibid., 1303 (1967). A complete list of earlier references may be found in Ref. 1.

3. R. Carlitz and M. Kislinger, Phys. Rev. D2, 336 (1970).

4. J. Rosner, Phys. Rev. Letters 22, 689 (1969).

5. L. Micu, Nuc1. Phys. B10, 521 (1969).

6. R.P. Feynman, M. Kislinger, and F. Ravndal, Phys. Rev. D3, 2706 (1971).

7. A.N. Mitra and R. Mehrotra, Phys. Rev. D4,'1409.(1971) and to be published.

8. D. Faiman and A.W. Hendry, Phys. Rev. 173, 1720 (1968).

9. D. Faiman, Nucl. Phys. B32, 573 (1971).

10: This sign change indeed occurs in Ref. 6.

11. A.D. Brody, et al., Phys. Lett. 34B, $665^{\circ}$ (1971); Phys. Rev. D4, 2693 (1971).

12. Jack Y.S. Sahouria, Thesis, LRL report UCRL-18624 (1969), unpublished.

13. J. Prevost, et al., presented by R. Barloutaud to Amsterdam International Conference on Elementary Particles, July, 1971.

14. W. Petersen and J. Rosner, in preparation.

15. M. Imachi, T. Matsuoka, K. Ninomiya, and S. Sawada, Prog. Theor. Phys. (Kyoto) 40, 353 (1968); H. Harari, Phys. Rev. Lett. 22, 562 (1969).

16. P.G.O. Freund, Nuovo Cimento 5A, 9 (1971).

17. L. Cook and G. Murtaza, Nuovo Cimento 39, 531 (1965).

18. Particle Data Group, Rev. Mod. Phys. 43, S1 (1971).

19. Parity conservation requires $a_{L}(1)=a_{L}(-1)$. 
20. A.R. Edmonds, Angular Momentum in Quantum Mechanics, Princeton University Press, 1960, p. 97.

21. A.H. Rosenfeld, private communication.

22. A. Kernan and H. Shepard, Phys. Rev. Lett. 23, 1314 (1969).

23. The $\Lambda(1830)\left(5 / 2^{-}\right)$is predicted to decouple from $\overline{K N}$ in the present model. The $\Sigma(1915)\left(5 / 2^{+}\right)$is outside the region of present high-statistics phase shift analyses.

24. Y. Gell, D. Horn, M. Jacob, and J. Weyers, CERN preprint TH-1346 (1971), to be published.

25. J. Rosner, in Proceedings of the Workshop on Particle Physics at Intermediate Energies, Mar. 29-30, 1971, ed. R.D. Field, Jr., LRL report UCRL-20655, p. 129. In this work the last line of. Eq. (43) should read $\Gamma\left(\mathrm{J}_{z}= \pm 3 / 2\right) / \Gamma\left(\mathrm{J}_{z}= \pm 1 / 2\right)=5 . \mathrm{SU}(6)_{\mathrm{W}}$ predicts no. $\mathrm{H}$ wave in $\Delta(1950) \rightarrow \Delta \pi$.

26. S. Mande1stam, Phys. Rev. D1, 1734 (1970).

27. S. Almehed and C. Lovelace, to be published.

28. C. Weil, Phỵs. Rev. 161, 1682 (1967).

Table I. Comparison of predicted partial widths of $70, \mathrm{~L}=1$ baryons with experiment. Data frim Refs. 18 and 27.

Table II. Approximate mixing scheme for $\Lambda\left(1 / 2^{-}\right)$. Table III. $\underline{56}, \mathrm{~L}=2$ baryons. Data from Refs. 18, 27. 
TABLE I

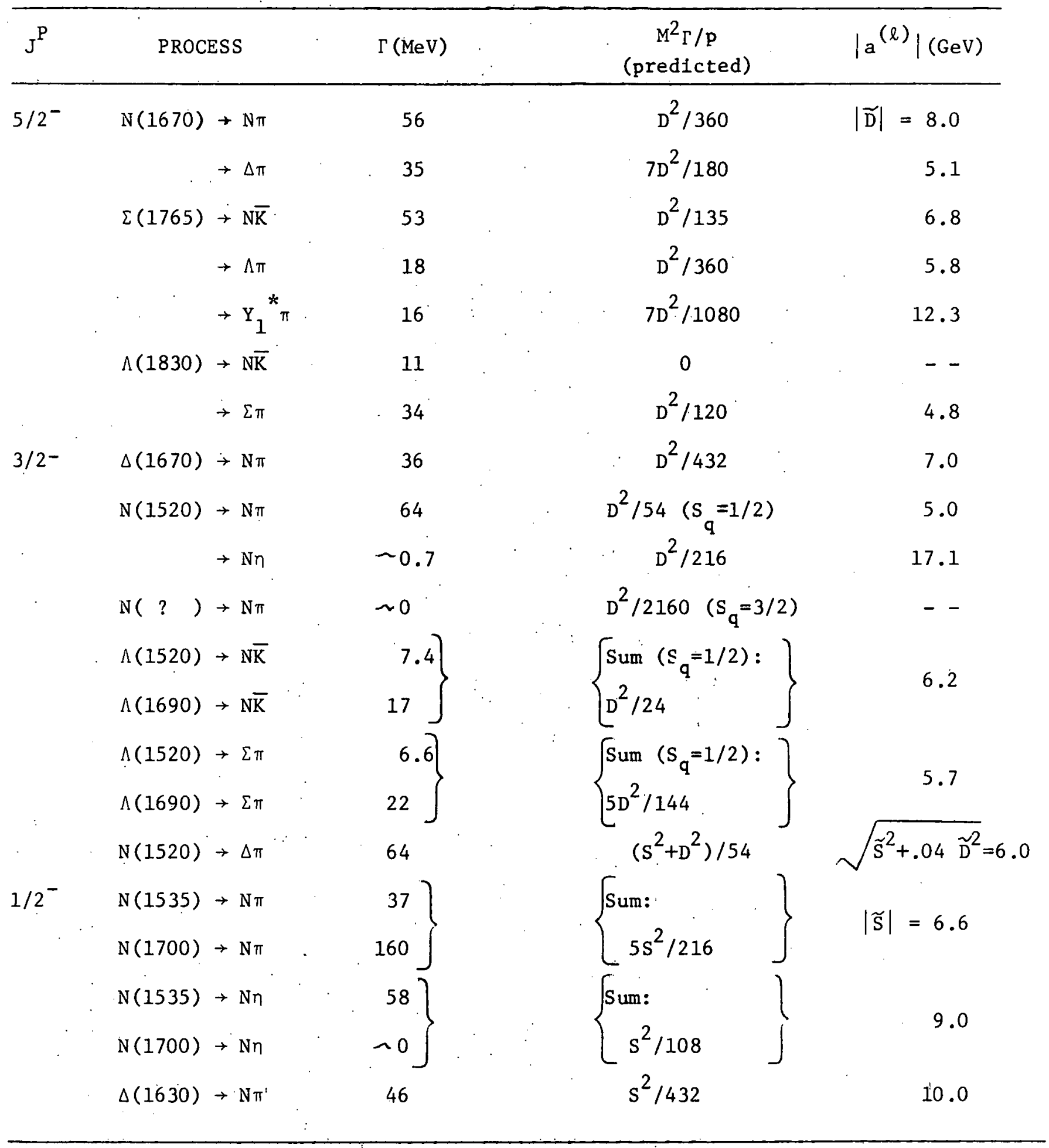


$-12-$

TABLE II

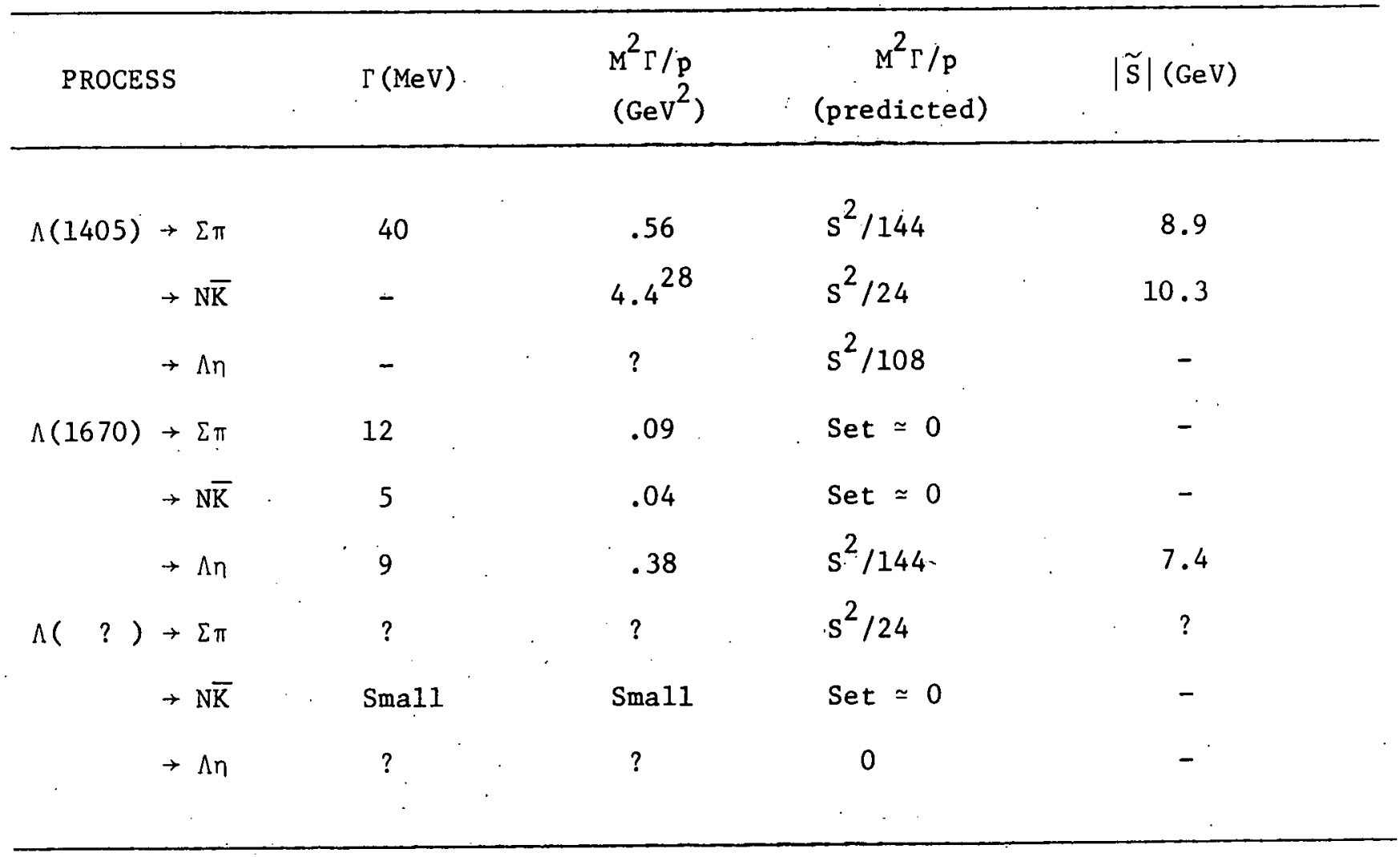


TABLE III

\begin{tabular}{|c|c|c|c|c|c|c|}
\hline$J^{P}$ & PROCES & & $\Gamma(\mathrm{MeV})$ & $\begin{array}{c}M^{2} \Gamma / p \\
\text { (predicted) }\end{array}$ & $\left|\tilde{a}^{(l)}\right|$ & | (GeV) \\
\hline \multirow[t]{6}{*}{$7 / 2^{+}$} & $\Delta(1950)$ & $\rightarrow \mathrm{N} \pi$ & 80 & $4 F^{2} / 35$ & $|\tilde{F}|=2$ & 2.3 \\
\hline & 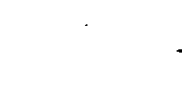 & $\rightarrow \Sigma \mathrm{K}$ & 4 & $4 \mathrm{~F}^{2} / 525$ & - & 2.7 \\
\hline & & $\rightarrow \Delta \pi$ & 115 & $\mathrm{~F}^{2} / 70$ & & 4.9 \\
\hline & $\Sigma(2030)$ & $\rightarrow \mathrm{N} \overline{\mathrm{K}}$ & 25 & $4 \mathrm{~F}^{2} / 1575$ & & 2.8 \\
\hline & & $\rightarrow \Lambda \pi$ & 35 & $2 F^{2} / 525$ & & 2.7 \\
\hline & & $\rightarrow \Sigma \pi$ & 5 & $4 \mathrm{~F}^{2} / 1575$ & & 1.5 \\
\hline \multirow[t]{7}{*}{$5 / 2^{+}$} & $N(1690)$ & $\rightarrow \mathrm{N} \pi$ & 91 & $F^{2} / 54$ & & 3.3 \\
\hline & $\Lambda(1815)$ & $\rightarrow \Sigma \pi$ & 9 & $2 F^{2} / 225$ & & 2.5 \\
\hline & & $\rightarrow \mathrm{N} \overline{\mathrm{K}}$ & 51 & $F^{2} / 75$ & . & 3.9 \\
\hline & $\sum(1915)$ & $\rightarrow \mathrm{N} \overline{\mathrm{K}}$ & 8 & $F^{2} / 2025$ & & 5.2 \\
\hline & $\Delta(1890)$ & $\rightarrow \mathrm{N} \pi$ & 45 & $8 F^{2} / 4725$ & & 4.2 \\
\hline & $N(1690)$ & $\rightarrow \Delta \pi$ & $18^{21}$ & $(16 / 3375)\left(2 F^{2}+3 P^{2}\right)$ & $\sqrt{\tilde{\mathrm{P}}^{2}+.20 \tilde{\mathrm{F}}^{2}}=$ & 4.1 \\
\hline & $\Lambda(1815)$ & $\rightarrow \mathrm{Y}_{1}^{*} \pi$ & 14 & $(4 / 1125) \quad\left(2 \mathrm{~F}^{2}+3 \mathrm{P}^{2}\right)$ & $\sqrt{\tilde{\mathrm{P}}^{2}+.17 \tilde{\mathrm{F}}^{2}}=$ & 4.8 \\
\hline \multirow[t]{2}{*}{$3 / 2^{+}$} & $N(1860)$ & $\rightarrow \mathrm{N} \pi$ & 75 & $\mathrm{P}^{2} / 54$ & $|\widetilde{\mathrm{P}}|=$ & 3.3 \\
\hline & & $\rightarrow \Lambda \mathrm{K}$ & 18 & $P^{2} / 150$ & & 5.3 \\
\hline $1 / 2^{+}$ & $\Delta(1910)$ & $\rightarrow \mathrm{N} \pi$ & 66 & $8 P^{2} / 675$ & 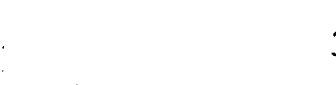 & 3.7 \\
\hline
\end{tabular}

